\title{
Mode locking of a broad-area semiconductor laser with a multiple-quantum-well saturable absorber
}

\author{
L. E. Adams and E. S. Kintzer \\ MIT Lincoln Laboratory, Lexington, Massachusetts 02173
}

M. Ramaswamy and J. G. Fujimoto

Department of Electrical Engineering and Computer Science, Massachusetts Institute of Technology, Cambridge, Massachusetts 02139

\author{
U. Keller*
}

ATET Bell Laboratories, Holmdel, New Jersey 07733

\author{
M. T. Asom
}

ATET Bell Laboratories, Breinigsville, Pennsylvania 18031

Received August 2, 1993

\begin{abstract}
Hybrid mode locking of a broad-area semiconductor laser with a multiple-quantum-well saturable absorber in an external cavity is demonstrated. A novel method for mode control of the broad-area laser output, based on patterning of the multiple quantum well absorber into a microdot mirror structure, is presented. Pulses as short as $15 \mathrm{ps}$, at a repetition rate of $593 \mathrm{MHz}$, with an average power of $9 \mathrm{~mW}$ and a peak power of $1 \mathrm{~W}$ have been achieved.
\end{abstract}

The highly efficient and compact nature of semiconductor diode lasers makes these sources attractive for generating short optical pulses. Active mode locking at high modulation frequencies can produce subpicosecond pulses. Pulses as short as $0.58 \mathrm{ps}$ at a 16-GHz repetition rate, with $30 \mathrm{~mW}$ of peak power and $0.5 \mathrm{~mW}$ of average power in the pulse burst, have been generated by active mode locking of a single-stripe diode in an external cavity. ${ }^{1}$ However, the power available from mode-locked single-stripe diodes is limited. More power can be made available for mode locking by use of large-area diode arrays or broad-area lasers. However, the large capacitance of broad-area lasers and diode arrays makes highfrequency modulation difficult, limiting the pulse widths that can be achieved from active mode locking.

Another method of generating short pulses is passive mode locking with a saturable absorber created by ion implantation of one diode facet. Pulses from a single-stripe diode laser have been externally compressed to durations as short as $230 \mathrm{fs}$ with average power of $2.3 \mathrm{~mW} .^{2}$ An ion-implanted diode array has produced 5-ps pulses with an average power of $400 \mathrm{~mW} .^{3}$ The spatial mode quality of the array output may restrict the applications of these pulses. Recently, some mode control has been demonstrated from this type of mode-locked array by the use of angularly selective optical feedback. Pulses as short as $3 \mathrm{ps}$ with an average power of $5.5 \mathrm{~mW}$ have been reported. ${ }^{4}$

It is useful to have a saturable absorber that is completely independent of the laser structure. This independence allows the absorber to be cus- tom designed to optimize saturation and recovery characteristics. Single-stripe diode lasers and semiconductor traveling-wave amplifiers have been passively mode locked in an external cavity by use of a multiple-quantum-well (MQW) saturable absorber. A diode laser has produced pulses as short as $1.6 \mathrm{ps}$ with average power of $1 \mathrm{~mW} .^{5,6} \mathrm{~A}$ traveling-wave amplifier system has yielded 5-ps pulses with an average power of $500 \mu \mathrm{W}$. The average power was increased to $800 \mu \mathrm{W}$ by hybrid mode locking. An external amplifier and compressor reduced the pulse width of these traveling-wave amplifier hybrid modelocked pulses to $207 \mathrm{fs}$ with a peak power of $165 \mathrm{~W} .^{7}$

It is desirable to have a source for generating highpower picosecond pulses that offers both simplicity and flexibility of design. In this Letter we present what is to our knowledge the first demonstration of a hybrid mode-locked broad-area semiconductor laser with an MQW saturable absorber in an external cavity. The absorber that we have used is custom tailored for our desired absorption and is grown under conditions that ensure a fast recovery time. Our external cavity is limited to only a few optical elements including an output coupler (OC), saturable absorber, and lenses.

Our saturable absorber, shown in the inset of Fig. 1 , is a low-temperature (LT) $\left(\approx 300^{\circ} \mathrm{C}\right)$ molecular-beam-epitaxy-grown AlGaAs-GaAs MQW structure with 38 periods of 10 -nm-thick $\mathrm{GaAs}$ quantum wells and 3.5-nm $\mathrm{Al}_{0.3} \mathrm{Ga}_{0.7} \mathrm{As}$ barriers. The LT MQW structure is grown on top of a $\mathrm{Al}_{0.3} \mathrm{Ga}_{0.7} \mathrm{As} / \mathrm{AlAs}$ dielectric mirror stack with 16 periods of 72.3-nm $\mathrm{AlAs}$ and 59.9-nm AlGaAs layers grown on a GaAs 

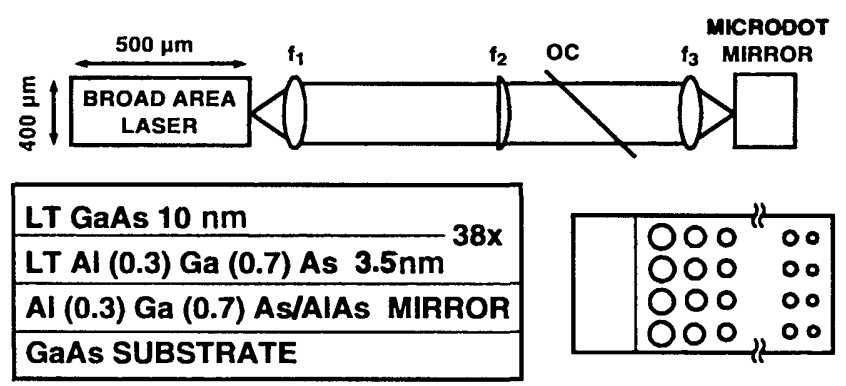

Fig. 1. Schematic of the hybrid mode-locked broad-area laser external cavity. The beam is shown perpendicular to the junction. The insets show the microdot pattern and the LT GaAs-AlGaAs quantum-well reflector.

substrate at normal growth temperature. The excitonic resonance occurs at $\sim 840 \mathrm{~nm}$. In other diode mode-locking experiments that used a MQW saturable absorber, the GaAs substrate was removed and the absorber was epoxied to a high-reflection mirror..$^{5-7}$ Here we have the ability to design the dielectric mirror and integrate it monolithically with the MQW saturable absorber. This approach was demonstrated previously for saturable absorber mode locking of solid-state lasers., ${ }^{8,9}$

Replacing a single-stripe diode laser with a broadarea semiconductor laser introduces the issue of mode control in order for light to be well focused onto the saturable absorber. The MQW sample is selectively etched into a microdot pattern with dots ranging in diameter from 30 to $5 \mu \mathrm{m}$. The external-cavity laser was designed to transform a highly asymmetric optical mode emitted from the broad-area laser into a small, nearly symmetric beam that could be focused onto the microdot. By translating the microdot mirror sample, we lase on increasingly smaller-diameter dots, which act as apertures, forcing the laser to operate in a few of the lowest-order modes.

According to Haus, ${ }^{10}$ stable mode locking requires that the loss both saturate and recover faster than the gain. Mathematically, these conditions may be stated as $\sigma_{A} / A_{A}>\sigma_{G} / A_{G}$ for the saturation cross section and $\tau_{A}<\tau_{G}$ for the recovery time, where $\sigma_{A}$ and $\sigma_{G}$ are the effective cross sections for the absorber and the gain, respectively, $A_{A}$ and $A_{G}$ are the geometrical cross sections of the laser beam in the absorber and the gain, and $\tau_{A}$ and $\tau_{G}$ are the recovery times of the absorber and the gain. $\mathrm{Be}-$ cause of the LT molcular-beam-epitaxy growth, which broadens the excitonic features, ${ }^{11}$ we cannot expect a much larger $\sigma_{A}$ than $\sigma_{G}$. However, we utilize area scaling to increase the normalized saturation cross section of the absorber relative to the gain. The beam size at the facet is comparable with the spot size on the microdot in the vertical dimension but is more than a factor of 15 times larger in the horizontal dimension, making $A_{G}$ much greater than $A_{A}$. Hence the first mode-locking condition given above is satisfied. Furthermore, area scaling acts to deemphasize the role that nonlinear gain dynamics play in limiting the minimum attainable pulse duration. ${ }^{7}$ Previously, proton bombardment and tight focusing methods were used to speed up the absorber recovery time in order to comply with the second condition stated above. ${ }^{5-7}$ In this research we rely on LT molecular-beam-epitaxy growth of the MQW saturable absorber. This creates defects that give rise to much faster carrier recombination, resulting in a significantly reduced recovery time for the saturable absorber. ${ }^{11}$

The microdot pattern and a schematic of the modelocked laser external cavity are shown in Fig. 1. The gain medium is a MQW broad-area semiconductor laser (manufactured by Spectra Diode Laboratories) operating at $830 \mathrm{~nm}$ with an active area $400 \mu \mathrm{m}$ wide and $500 \mu \mathrm{m}$ long. The front facet was antireflection coated, $R \sim 2 \%$, and the back facet was high-reflection coated. The laser had a threshold of 1.3 A. To map the highly asymmetric laser output onto the microdot, we used $A B C D$ matrix techniques to calculate spot sizes and stability regions. In the vertical dimension, the objective lens, $f_{1}=8.6 \mathrm{~mm}$, and the focusing lens, $f_{3}=8 \mathrm{~mm}$, form a telescope. In the horizontal dimension, the cylindrical lens, $f_{2}=$ $200 \mathrm{~mm}$, acting in combination with the objective lens, functions to fill the aperture of the focusing lens.

The MQW sample is divided into an unpatterned region and a region containing the microdots, as shown in Fig. 1. At our operating wavelength of $830 \mathrm{~nm}$, we measured a $42 \%$ reflectivity from the unpatterned portion of the MQW. In this experiment, we achieved the best results by lasing on an $\sim 13-\mu \mathrm{m}$ diameter dot. The smaller-diameter dots on our sample were damaged by the etching process and did not produce stable mode-locked pulses. The largest dots provided insufficient mode control, resulting in pulses comparable with those obtained with the unpatterned MQW. The MQW sample is mounted onto an $\sim 60 \%$ reflectivity aluminum mirror. The external-cavity laser had $\mathrm{cw}$ threshold currents of 1.15 and 1.2 $\mathrm{A}$ when it was lasing on the mirror and on the microdot, respectively.

A modulated injection current was passed through a rf amplifier and applied to the broad-area laser through an impedance-tuning network and a bias tee. The rf was $593 \mathrm{MHz}$, corresponding to the second harmonic of the external cavity. A $R=10 \%$ pellicle beam splitter was placed in the cavity to function as an output coupler. We viewed the beam at the end mirror by using reflected light from one surface of the beam splitter and imaging the plane of the mirror onto a camera. Output light from the other pellicle surface was directed to a second-harmonic autocorrelator. A second pellicle could be inserted in the cavity to image the laser facet. Depending on alignment, lasing would occur across one half to two thirds of the facet.

Mode locking was observed when the laser beam was focused onto the aluminum mirror, the unpatterned MQW, or the microdot. Figure 2 shows second-harmonic autocorrelation traces of the shortest pulses obtained from each of the three reflectors. Active mode locking with the $60 \%$ reflectivity mirror yielded pulses as short as 51 ps, as shown in Fig. 2(a). Hybrid mode locking with the unpatterned MQW reduced the pulse width to $37 \mathrm{ps}$, as shown in Fig. 2(b), owing to the saturable absorber action. 


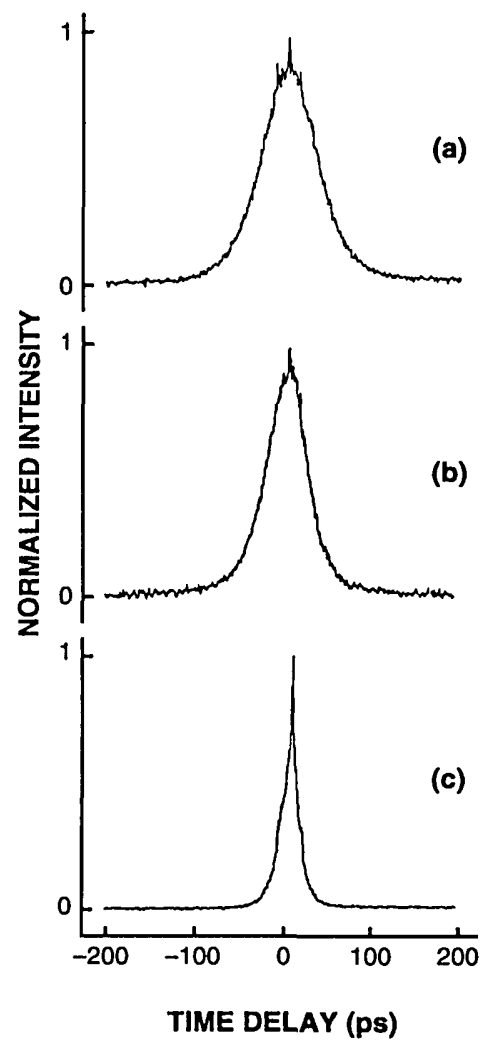

Fig. 2. Autocorrelation traces of mode-locked laser output pulses that were generated with (a) the aluminum mirror, (b) the unpatterned MQW, and (c) the microdot.

Enhanced mode control, provided by the microdot, produced better saturable absorption, resulting in further pulse shortening, as shown in Fig. 2(c). Hybrid mode locking with the microdot produced 15-ps pulses, at a repetition rate of $593 \mathrm{MHz}$, with an average power of $9 \mathrm{~mW}$ and a peak power of $1 \mathrm{~W}$. In calculating these powers, we have summed the output intensities of the light reflected from the front and back surfaces of the output coupling beam splitter. Residual reflectivity of the antireflectioncoated laser facet resulted in coherence spikes, which were separated by the round-trip time of the broadarea laser. All pulse durations were measured with the amplitude of the coherence spike neglected. The observed spectral width corresponding to the 15-ps pulse was $1.35 \mathrm{~nm}$, indicating a time-bandwidth product of 8.1 , which is $\sim 18$ times the Fouriertransform limit. Since the laser operates in many longitudinal modes of the diode cavity, we suspect that the time-bandwidth product could be reduced by bandwidth limiting. Improved system performance should also be attainable by application of a better antireflection coating to the laser facet. In addition, we were not able to tune our laser to the center of the excitonic resonance, where we would expect to see further pulse shortening.

In summary, we have demonstrated a simple, compact system for mode locking a broad-area laser, which generates picosecond pulses at higher powers than those attainable with single-stripe diodes. Mode control was shown to be an essential element for producing short pulses with a broad-area laser. We have presented a novel method for achieving this mode control that is based on an MQW microdot mirror structure. Our saturable absorber was independent of the laser and offered custom tailored saturation and recovery characteristics. Pulses as short as 15 ps with an output peak power of $1 \mathrm{~W}$ were achieved.

This research was sponsored by the U.S. Department of the Air Force, Joint Services Electronics Program contract DAAL 03-91-C-0001, and National Science Foundation contract ECS-85-52701.

${ }^{*}$ Present address, Swiss Federal Institute of Technology, ETH Honggerberg, Zürich, Switzerland.

\section{References}

1. S. Corzine, J. Bowers, G. Przybylek, U. Koren, B. Miller, and C. Soccolich, Appl. Phys. Lett. 52, 348 (1988).

2. N. Stelmakh and J.-M. Lourtioz, Electron. Lett. 29, 160 (1993).

3. J. Zarrabi, E. L. Portnoi, and A. V. Chelnokov, Appl. Phys. Lett. 59, 1526 (1991).

4. A. Chelnokov, J.-M. Lourtioz, and P. Gavrilovic, Electron. Lett. 29, 861 (1993).

5. Y. Silberberg, P. W. Smith, D. J. Eilenberger, D. Miller, A. Gossard, and W. Wiegmann, Opt. Lett. 9, 507 (1984).

6. P. W. Smith, Y. Silberberg, and D. Miller, J. Opt. Soc. Am. B 2, 1228 (1985).

7. P. Delfyett, L. Florez, N. Stoffel, T. Gmitter, N. Andreadakis, Y. Silberberg, J. Heritage, and G. Alphonse, IEEE J. Quantum Electron. 28, 2203 (1992).

8. U. Keller and T. Chiu, IEEE J. Quantum Electron. 28, 1710 (1992).

9. U. Keller, W. Knox, and G. 'tHooft, IEEE J. Quantum Electron. 28, 2123 (1992).

10. H. Haus, IEEE J. Quantum Electron. QE-11, 736 (1975).

11. W. Knox, G. Doran, M. Asom, G. Livescu, R. Leibenguth, and S. Chu, Appl. Phys. Lett. 59, 1491 (1991). 